\title{
FAKTIZITÄTEN
}

\section{Einleitung in den Schwerpunkt}

1 Letzterer Begriff geht zurück auf die satirische Late-Night-Show von Stephen Colbert: The Colbert Report, Comedy Central, USA 2005-2014.

2 Vgl. z. B. die Fact-CheckAbteilung der New York Times (nytimes.com/spotlight/fact-checks) oder die gemeinnützige Rechercheplattform Correctiv (correctiv.org), gesehen am 18.7.2018.

3 Vgl. Harry G. Frankfurt: On Bullshit, Princeton 2005.

4 Vgl. Ulrich Ladurner: Stadt der Lügner, in: Die Zeit, Nr. 52, 18.12.2016, online archiviert unter hdl.handle.net/11346/KWZW, gesehen am 18.7.2018.

5 Neil MacFarquhar: Inside the Russian Troll Factory: Zombies and a Breakneck Pace, in: The New York Times, dort datiert 18.2.2018, nyti. $\mathrm{ms} / 2 \mathrm{C} 6 \mathrm{RZoE}$, gesehen am 18.7.2018.

6 Vgl. z. B. The Guardian: The Cambridge Analytica Files, online unter theguardian.com/news/series/ cambridge-analytica-files, gesehen am 20.6.2018.

7 Vgl. Bernhard Pörksen: Die große Gereiztheit: Wege aus der kollektiven Erregung, München 2018, 50.

8 Vgl.z. B. Eli Pariser: Filter Bubble: Wie wir im Internet entmündigt werden, München 2012.

\section{Sie haben Ihre Zahlen, ich habe keine}

Das Thema Faktizitäten verweist einerseits auf einen aktuellen gesellschaftlichen Diskurs und ist andererseits mit einer langen und weitreichend verzweigten Begriffsgeschichte verknüpft. Beides kann im Rahmen einer einzelnen Zeitschriftenausgabe nur unzureichend abgebildet werden. Der vorliegende Schwerpunkt macht es sich daher zur Aufgabe, den vielschichtigen Diskurs um Faktizität aus einer spezifisch medienwissenschaftlichen Perspektive in den Blick zu nehmen und damit die zu beobachtende Omnipräsenz von Schlagwörtern wie <Fake News>, <alternativen Fakten> oder <truthiness> kritisch zu befragen. ${ }^{1}$ Angesprochen ist damit ein Diskurs(schlacht-)feld, das sich zwischen verbissenem Fact-Checking ${ }^{2}$ und faktenindifferentem Bullshitting ${ }^{3}$ aufspannt. Umtost von den Kommunikations- und Aufmerksamkeitsdynamiken digitaler Plattformen, in denen die Portabilität, das leichte Sharing und die Veränderbarkeit digitaler Daten zu ökonomischen Schmetterlingseffekten führten, diffundiere zunehmend die bewusste Unterscheidung von Fakt und Fake mittels gezielt platzierter, 〈fabrizierter> Informationen aus den Heimwerkstätten, ${ }^{4}$ Fabriken ${ }^{5}$ oder Start-ups ${ }^{6}$ der Meinungstrollerei, so die einen. Die Berufung auf Fakten, hält die andere Seite dagegen, sei heute nicht mehr den sogenannten Qualitätsmedien vorbehalten: Den etablierten Nachrichtenmedien wird vorgeworfen, sich von einer korrupten Politikerelite instrumentalisieren zu lassen und Fakten beliebig zu manipulieren. Indem beide Seiten dasselbe Argument anführen, nämlich die jeweiligen Gegner des Fakes zu bezichtigen und gleichzeitig selbst die Wahrheit bereits zu besitzen, wird eine klassische, faktenbasierte Wahrheitssuche diskreditiert. Stattdessen, so wiederum Beobachter dieses Diskurses, entstehe ein neuer Sofortismus ${ }^{7}$ der unmerklichen Manipulation durch Social Bots und der algorithmischen Verzerrung von 
Meinungsbildungsprozessen durch individualisierende Echokammer-Effekte ${ }^{8}$ im Hintergrund, welcher verschiedentlich als Heraufdämmern einer «postfaktischen Ära» ${ }^{9}$ beschrieben und institutionell beglaubigt wurde. ${ }^{10}$ Dieses neue Zeitalter sei gekennzeichnet durch einen grundsätzlichen Skeptizismus gegenüber möglichen Zugängen zu einer <objektiven Realität> und allgemeingültigen Unterscheidungen zwischen 〈wahr $>$ und $\langle$ falsch $>$.

Die damit heraufbeschworenen Dynamiken des ständigen Meinens (und dessen unablässiger Distribution), aber auch der bewussten Fabrikation von Falschmeldungen und Lügen sollen einerseits vermehrt durch koordinierte crackdowns eingehegt werden - meist mittels politisch nicht unheikler Eingriffe in netzbasierte <Informationsfreiheiten>." Andererseits reagieren Teile des akademischen Bereichs weltweit mit Science Marches samt seltsam positivistischer Schnellschüsse à la 〈Zu Fakten gibt es keine Alternative! > auf den genannten Zweifel an Faktizitäten. Letztere appellierten dabei jedoch an eine «Szientokratie», so etwa Peter Strohschneider in einer differenzierten Auseinandersetzung mit gegenwärtigen Wahrheitskonstruktionen im Spannungsfeld von Politik, Wissenschaft und Medien, die «ihrer inneren Logik nach antipluralistisch» sei - genau wie die Autokrat_innen und Populist_innen, gegen die sie sich wende. ${ }^{12}$

In den Blick gerät daher zunehmend die wissenschaftstheoretische $\mathrm{Na}$ ivität von Post-Trutb: War Wahrheit je ein beherrschendes Regulativ von Politik oder gar medialer Berichterstattung? Behauptet der Begriff nicht einen Zugang zu Fakten, den er anderen im Umkehrschluss abspricht? Impliziert er nicht die Möglichkeit zur Erkenntnis <objektiver> Wahrheiten, die alles wissenschaftshistorisch belegte Zweifeln an Faktizitäten quer durch verschiedenste Disziplinen ignoriert? ${ }^{13}$ Und verstellt dies nicht gerade den Blick auf die viel gefährlichere Verschiebung, die Wahrheit zur Funktion von Macht degradiert?

\section{Faktizitäten}

Der Wissenschaftshistorikerin Lorraine Daston zufolge können wir auf «Institutionen, die für Tatsachen garantieren, nicht verzichten.» Wahrheit komme in der Regel nicht durch «Geniestreiche ans Licht», sondern müsse «mühsam erarbeitet werden - und dazu tun Institutionen, Methoden und Beweismittel not $\gg^{14}-$ Möglichkeitsbedingungen, die auch BENJAMIN PETERS in seinem Beitrag diskutiert. Dabei weist Daston auf die vertrackte Genese des Fakten-Begriffs hin: Noch in der Frühneuzeit war der Aspekt des Hergestellten und Gemachten parallel zur Vorstellung von Fakten als (göttliche) Gegebenheiten verbreitet. Erst mit dem Objektivitätsanspruch moderner (Natur-)Wissenschaften, der z. B. durch Prinzipien der Wiederholbarkeit, des Ausschlusses <unwissenschaftlichen> Imaginierens durch die Einbindung technischer Geräte und des <kollektiven Empirismus〉 neugeschaffener
9 Vgl. z. B. Ralph Keyes: The Post-Truth Era, New York 2004.

$10 \mathrm{Vgl}$. Oxford Dictionaries: Word of the Year 2016 is..., en.oxforddictionaries.com/word-ofthe-year/word-of-the-year-2016, gesehen am 20.6.2018.

11 Vgl. z. B. N.N.: First Person Convicted under Malaysia's Fake News Law, in: The Guardian, dort datiert 30.4.2018, theguardian.com/ world/2018/apr/3o/first-personconvicted-under-malaysias-fake-newslaw, gesehen am 20.6.2018; N.N.: EU Anti-propaganda Unit Gets $€ 1 \mathrm{M}$ a Year to Counter Russian Fake News, in: The Guardian, dort datiert 25.11.2017, theguardian.com/world/ 2017/nov/25/eu-anti-propaganda-unitgets-1m-a-year-to-counter-russianfake-news, gesehen am 20.6.2018.

12 Peter Strohschneider: Über Wissenschaft im Zeitalter des Populismus, Rede auf der Jahresversammlung der DFG am 4.7.2017, online unter $\mathrm{dfg}$.de/download/pdf/dfg_im_pro fil/reden_stellungnahmen/2017/170704rede_strohschneider_festueranstaltung. pdf, gesehen am 20.6.2018. Vgl. auch Armin Nassehi: Zu Fakten gibt es oft eine Alternative, in: Frankfurter Allgemeine Zeitung, 28.6.2017, N 4; Aktuelle Publikationen zum Themenfeld umfassen u. a. Melissa Zimdars, Kembrew McLeod (Hg.): Fake News. Understanding Media and Misinformation in the Digital Age, Cambridge 2018; Zeitschrift für Medienphilosophie und Kulturtechnikforschung, Nr. 9/2, 2018: Alternative Fakten (in Vorbereitung); Günter Blamberger, Axel Freimuth, Peter Strohschneider (Hg.): Vom Umgang mit Fakten. Antworten aus Natur-, Sozial- und Geisteswissenschaften, Paderborn 2018; Karsten Könnecker (Hg.): Fake oder Fakt? Wissenschaft, Wahrheit, Vertrauen, Berlin, Heidelberg 2018; Daniel J. Levitin: Weaponized Lies: How to Think Critically in the Post-truth Era, New York 2017; Brian McNair: Fake News: Falsehood, Fabrication and Fiction in Journalism, London, New York 2017; Bruce McComiskey: Post-Truth Rhetoric and Composition, Boulder 2017; Michael A. Peters u. a.: Post-Truth, Fake News: Viral Modernity \& Higher Education, Springer 2018.

13 Vgl. Pörksen: Die große Gereiztheit, $40 \mathrm{f}$.

14 Philipp Felsch, Lorraine Daston, Georg Mascolo: Welchen Fakten können wir trauen?, in: Philosophie Magazin, Nr. 3, 2017, 62. 
15 Vgl. Lorraine Daston: Fear and Loathing of the Imagination in Science, in: Daedalus, Vol.134, Nr. 4, 1998, 16-30, hier 18. Vgl. auch dies., Peter Galison: Objektivität, Frankfurt/M. 2017. Zur Differenzierung von Daten und Fakten vgl. z. B. Daniel Rosenberg: Data before the Fact, in: Lisa Gitelman ( $\mathrm{Hg}$.): Raw Data is an Oxymoron, Cambridge, London 2013, 15-40.

16 Vgl. François Raffoul, Eric Sean Nelson: Introduction, in: dies. (Hg.): Rethinking Facticity, Albany 2008, 2-4.

17 Jean-Luc Nancy: Das Vergessen der Philosophie, Wien 2010, 96.

18 Vgl. Martin Doll: Fälschung und Fake. Zur diskurskritischen Dimension des Täuschens, Berlin 2015. wissenschaftlicher Akademien gesichert werden sollte, drehte sich diese <konstruktive Arbeit $>$ in analytische Verfahren um, die sich mehr dem Schmelzen und Säubern (smelting and purifying) als dem Bauen und Konstruieren (building and constructing) verschrieben, so Daston. Zunehmend delegiert an die <technische Objektivität> von Apparaturen, Aufzeichnungsmedien, und Experimentalsystemen, sorgten diese Verfahren dafür, Fakten als «naturgegebene> Unumstößlichkeiten erkennbar zu machen. ${ }^{15}$ Während sich Theorien und sogar die Objektivität selbst dem immer schnelleren Wandel der Zeit ausgesetzt sähen, bildeten 〈harte Fakten〉 ein Residuum überzeitlicher Gültigkeit. Ein derartiger Empirismus und Positivismus provozierte jedoch immer wieder auch heftige Auseinandersetzungen - mit den <Wahrheitskriegen $>$ der I 99oer Jahre und aktuellen Kontroversen rund um den 〈Spekulativen Realismus〉, die in den Beiträgen von BERNHARD PÖRKSEN und CLAUS PIAS thematisiert werden, als rezenten Ausprägungen.

Auch philosophische Begriffsgenealogien betonen die Bedeutungsvielfalt der Begriffe Faktizität und Fakt: So wirkte Gianbattista Vicos frühmoderner Begriff des verum factum als menschgemachter Tat bis in den deutschen Idealismus und in den Marxismus nach. Und selbst als das empiristisch-positivistische Ringen um objektive wissenschaftliche Wahrheiten mehr und mehr in den Vordergrund rückte, wurden philosophische Fragen nach dem Status von - hier hilft der differenzierende Sprung ins Englische - facticity im Gegensatz zur empirischen factuality weiterhin diskutiert: Von Kants tranzendentalem Faktum der Vernunft oder Diltheys Tatsächlichkeit über Heideggers frühes Projekt einer Hermeneutik der Faktizität hin zu Merleau-Pontys phänomenologisch-essenzialistischem Verständnis und zu Habermas' Dualismus von Faktizität und Geltung. ${ }^{16}$ Für Jean-Luc Nancy schließlich geht die Faktizität dieser Welt insofern mit deren Bedeutung einher, als dass das «Element des Sinns eine Realität» sei, «die ununterscheidbar und zugleich empirisch und transzendental, materiell und ideell, physisch und geistig ist - eine ganz neuartige <Vernunfttatsache>, die gleichzeitig die Reinheit einer Logik und die Festigkeit eines Fleisches besäße». ${ }^{17}$

Das in diesem Themenschwerpunkt vorgeschlagene Verständnis von Faktizitäten schließt hier an, indem es die medialen Operationen in den Blick nimmt, welche die bei Nancy genannten Simultanitäten prozessieren. Der Begriff eröffnet eine Heuristik, die eine kritische Diskussion der Bedingungen, der Bestimmungen und möglicher Folgen von <Fakten〉 in Bezug auf aktuelle mediale Praktiken, Techniken, Politiken und Ästhetiken sowie deren Schnittstellen ermöglicht. In den Blick geraten dabei neben den $<$ Fakten $>$ immer auch deren <Alternativen〉, die Rede über Faktizitäten schließt die Rede über Fälschung und Fake ${ }^{18}$ mit ein. Die Unterscheidung von Fälschung und Fake liegt dabei Martin Doll zufolge darin, dass Fälschungen nur so lange funktionieren, wie sie nicht erkannt respektive benannt werden. Wird eine Fälschung aufgedeckt, ist ihre Grundbedingung, nämlich sich an die Stelle 
eines Originals zu setzen, zerstört. ${ }^{19}$ Fakes hingegen, so Doll, tragen das «Moment der Enthüllung» bereits mit sich. Zumindest in der deutschen Verwendung des Begriffs sei die «Enttäuschung» anders als bei Fälschungen nicht «akzidentiell, sondern [...] konstitutiv». ${ }^{20}$ Sowohl der Fälschung als auch dem Fake assistiert er jedoch die Fähigkeit, diskurskritisch wirksam zu werden. Nicht nur lenkten sie den Blick auf diskursive Aussage- und Möglichkeitsbedingungen, sondern brächten einen «grundlegenden Dissens gegenüber vorgefundenen Ordnungen, wie Wissen verteilt, oder allgemein, wie regiert wird, zur Artikulation». ${ }^{21}$

Fakten, sowohl in ihrer primären Erscheinung als auch in ihrer Falsifizierung, erscheinen je schon als ein relationales Gefüge, das sich aktuell jedoch vor allem in einer dem hypothetischen Index distribuierter digitaler Prozesse geschuldeten epistemologischen wie soziopolitischen Unübersichtlichkeit ausprägt - in einer fehlenden, dem menschlichen Verstehen zugänglichen «Ästhetik des Sublimen»22 für die Effekte der Verschränkung großer Datenmengen, multikausaler Relationen und automatisierter algorithmischer Prozesse. Der Plural Faktizitäten ist hier also bewusst gewählt: Mit ihm sei angezeigt, dass Medien Faktizität längst über ihre philosophisch-ontologischen Bestimmungen hinaus flexibilisieren, wenn ihr Operieren im Anschluss an gesicherte wie ungesicherte Faktenlagen Fakten schafft und sie sich an der Produktion, Dekonstruktion oder Transformation von Fakten beteiligen. Und zugleich ermöglicht der Begriff Faktizitäten eine historische Bewertung der angeblichen Disruptionseffekte dieser Verschränkung, wenn ein Zweifel an hergebrachten Mechanismen der Wahrheitsproduktion und des Wahrheitsdiskurses als typische Signatur jeweils neuer Medien in den Blick gerät - z. B. des Buchdrucks ${ }^{23}$ oder der Penny Press. ${ }^{24}$

\section{Techniken/Ästhetiken: Justice 4 Grenfell}

Am I4. Juni 2017 geriet inmitten des Londoner Stadtteils Kensington eine als Grenfell Tower bekannte Sozialwohnungsanlage aufgrund eines defekten Kühlschranks in Brand. Innerhalb von Minuten standen ganze Etagen und komplette Teile der Fassade in Flammen. Mehr als 70 Menschen starben, und schnell wurde die Katastrophe zu einem Sinnbild bürokratischen Versagens und zynischer Immobilienpolitik: Notfallsysteme waren defekt oder fehlten komplett, das Material der Fassade sprach Brandschutzvorschriften Hohn, und Hinweise auf Mängel von Bewohner_innen des Hochhauses waren im Vorfeld der Katastrophe von den Behörden ignoriert worden. ${ }^{25}$ Und selbst über ein Jahr später wartete ein Großteil der meist sozial benachteiligten ehemaligen Bewohner_innen noch immer auf die ihnen zugesicherten Ersatzquartiere im näheren Wohnumfeld (Abb. I und 2).

An der Aufarbeitung dieses Falles beteiligte sich auch die 20 I I vom israelischen Architekten Eyal Weizman an der Goldsmiths University in London
19 Vgl. ebd., 21.

20 Ebd., 24.

21 Ebd., 13.

22 Nick Srnicek: Navigating Neoliberalism: Political Aesthetics in an Age of Crisis, Vortrag auf der Tagung The Matter of Contradiction: Ungrounding the Object, Vassivière, 8.-9.9.2012.

23 Vgl. z. B. Felsch, Daston, Mascolo: Welchen Fakten können wir trauen, 60.

24 Vgl. z. B. Kevin Young: Bunk: The Rise of Hoaxes, Humbug, Plagiarists, Phonies, Post-facts, and Fake News, Minneapolis 2017.

25 Vgl. Jörg Schindler: Die toten Häuser von London, in: Der Spiegel, Nr. 24, 2018. 


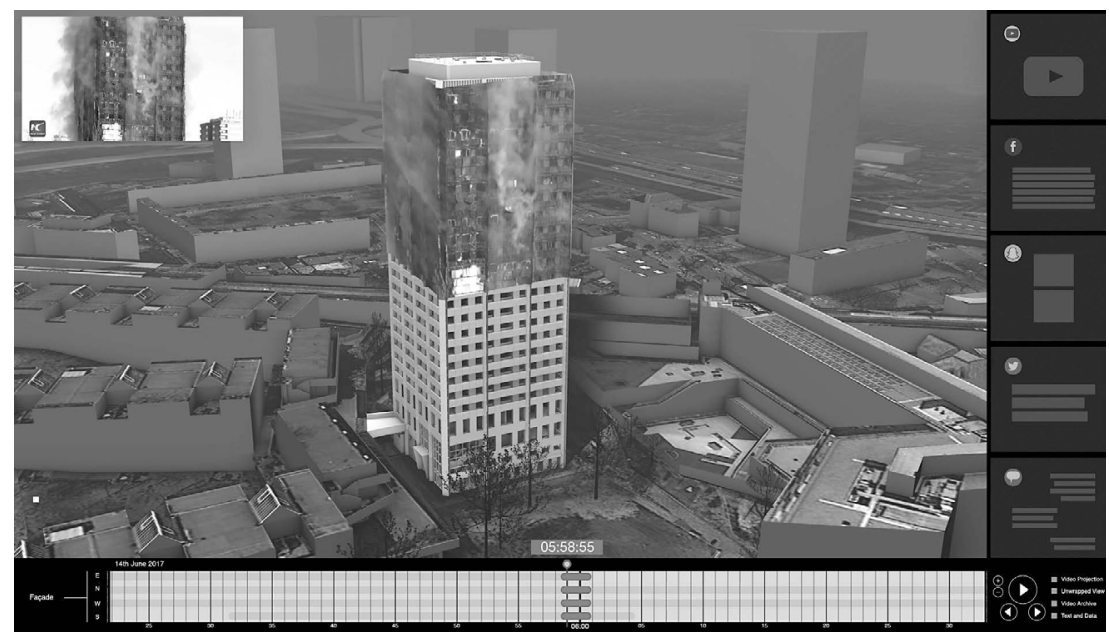

Abb. $1 / 2$ oben Prototyp für ein ${ }_{3}$ D-Video zur Analyse des Brands: Mapping und Projektion von Videomaterial auf ein Modell des Grenfell Tower mit navigierbarer Zeitleiste und Zugriff auf dokumentierte Kommunikationsvorgänge.

unten Alternative Ansicht mit perspektiviertem Videomaterial (texture projection). Bilder: Forensic Architecture, 2018

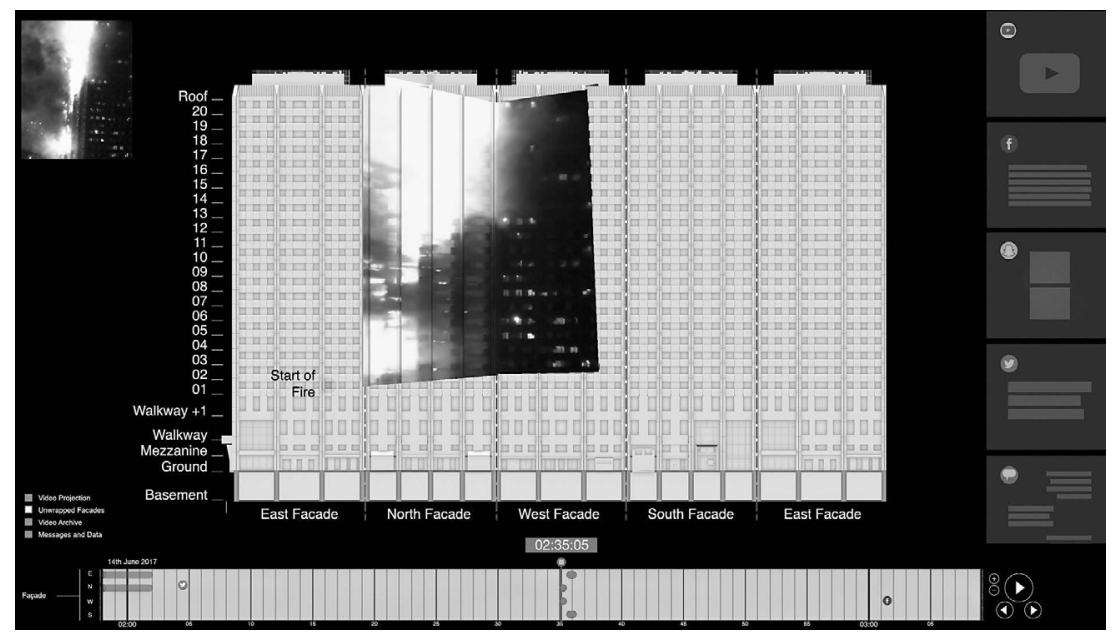

26 Der Begriff ist entlehnt von Allan Sekula: Photography and the Limits of National Identity, in: Culturefront, Vol. 2, Nr. 3, 1993, $54 \mathrm{f}$. Er wird ausführlich thematisiert in Thomas Keenan: Counter-Forensics and Photography, in: Grey Room, Vol. 55, 2014, 58-77. Die Arbeit der Agentur erlangte durch eine Reihe von TV-Berichten und durch Ausstellungen auf der documenta 14, der 15. Architekturbiennale Venedig 2016 oder im ICA London Bekanntheit. gegründete Agentur Forensic Architecture. Mit ihren unter dem Begriff der <counter-forensics> bekannt gewordenen Methoden macht sie sowohl auf politischer als auch künstlerischer Bühne Furore. ${ }^{26}$ Als Zusammenschluss von Architekt_innen, Filmemacher_innen, Programmierer_innen, Künstler_innen und Anwält_innen rekonstruiert die Agentur mit aufwendigen dreidimensionalen Computermodellen <uneindeutige> Szenarien aus Krisenregionen oder Fälle von Menschenrechtsverletzungen, die sich gegen die offizielle Darstellung von Vorgängen z.B. seitens staatlicher Akteur_innen und Institutionen richten - oder kurz: Sie nutzen «technology to expose injustice».27 <Counter-forensics> ist dabei als kollaboratives Verfahren konzipiert: Die Modellierungen von Forensic Architecture verdichten die Mannigfaltigkeit disperser Daten - z. B. Videos, Fotos, Audioaufzeichungen, Zeugenaussagen, 
Social-Media-Postings - und machen sie in Zeit und Raum lokalisierbar und explorierbar. Erst durch das cross-referencing partikularisierter Daten aus verschiedensten Quellen und unterschiedlichsten Formaten und ihrer Zusammenführung verdichten sich diese zu Fakten: «The only way to make sense of them is to locate them in space and time. You need to be able to see the relationship between evidence and space», so Weizman. ${ }^{28}$

Damit arbeitet Forensic Architecture an einer wirksamen Verschränkung von Medientechniken und -ästhetiken, deren aktuelle Dimensionen etwa von Alexander R. Galloway befragt wurden, der sich in seinem Buch The Interface Effect mit Möglichkeiten der adäquaten Repräsentierbarkeit von Daten auseinandersetzt, die prinzipiell «no necessary visual form» haben. ${ }^{29}$ In eine ähnliche Richtung gehen Überlegungen von Nick Srnicek. Am Beispiel globaler Finanzmärkte fordert er die Entwicklung von «suitably complex and abstract form $[\mathrm{s}]$ of aesthetic representation in order to modulate our access to it.» ${ }^{\mathbf{3 0}}$ Neben den dort aufgeführten Beispielen für Daten-(Re-)Präsentationen aus dem meist künstlerischen Bereich (etwa von Marc Lombardi über das Bureau d'Études hin zu Ryoji Ikeda), die eine <Gestalt〉 sozioökonomischer Strukturen und Effekte erscheinen lassen und eine Kritik gemeinhin sublimer, unfassbarer und <alternativloser> Gegenstandsbereiche eröffnen, wirken die explorationsfähigen Modelle von Forensic Architecture jedoch ungleich <aktiver>. Sie ermöglichen eine umfassende Konkretisierung abstrakter Datenkonvolute als virtuelle Verräumlichungen in architektonischen Computersimulationsmodellen: ein Zusammenspiel von «matter, media and memory» ${ }^{31}$ als eine Art entsubjektiviertes «cognitive mapping» im Sinne Jamesons. ${ }^{32}$

Im Fall Grenfell richtete die Agentur eine Plattform ein, auf die Bürger_ innen eigenes Material hochladen können und deren Software Schnittstellen anbietet, die es freiwilligen Expert_innen z.B. für ${ }_{3}$ D-Mapping oder Bildanalyse ermöglicht, an der Modellierung mitzuarbeiten. Man könnte das Ergebnis eine «open-source-investigation ${ }^{33}$ mit $<$ Citizen science>-Elementen nennen. Diese macht exemplarisch erkennbar, wie sich innerhalb einer umfassenden Digitalität erstens (keineswegs <rohe>, sondern je schon formatierte) Daten erst durch ihre medientechnisch-ästhetische Kontextualisierung zu Fakten synthetisieren und wie diese Faktizität zweitens im Zuge von juristischen Verfahren Geltung erlangen kann. ${ }^{34}$ Was dabei interessiere, so Weizman, sei gerade die Betrachtung jenes Schmutzes, den herkömmliche Ermittlungsmethoden im Sinne einer <klaren> Beweisführung und eines Herunterbrechens von Ereignissen auf einfache juristische Sachverhalte stets zu bereinigen suchten. <Counter-forensics〉 sei ein Verfahren zur Rekonstruktion der Vieldimensionalität von Ereignissen, in der allein deren weiterer politischer Zusammenhang deutlich werde ${ }^{35}$ - oder anders: eine Synthese von Daten zu Fakten, die einer tiefergehenden Analyse und damit weiteren Faktizitäten vorausgeht. Ganz getreu Hito Steyerls Neudenken von Giambattista Vicos Prinzip des verum factum als factum verum stellt sich Wahrheit in der
27 Adam Branson: Forensic Architecture: Using Technology to Expose Injustice, in: The Architect's Journal, 26.4.2018, hdl.handle.net/11346/XFGC, gesehen am 18.7.2018.

28 Ebd.

29 Alexander R. Galloway: The Interface Effect, Cambridge 2012, 81.

30 Vgl. Srnicek: Navigating Neoliberalism.

31 Edwin Heathcote: How Forensic Architecture is Using Technology to Uncover Injustice, in: British GQ Magazine, 28.4.2018.

32 Frederic Jameson: Cognitive Mapping, in: Marxism and the Interpretation of Culture, hg. v. Lawrence Grossberg u. Cary Nelson, Chicago 1990, 347-36o.

33 Heathcote: Forensic Architecture.

34 Vgl. Jürgen Habermas: Faktizität und Geltung, Frankfurt/M. 1997.

35 Vgl. Mladen Gladic: Schmutz

im Licht, in: Der Freitag, Nr. 24, 2018. 
36 Vgl. Hito Steyerl: Ungeschaffene Wahrheit. Produktivismus und Faktographie, dort datiert März 2009, eipcp.net/transuersal/0910/ steyerl/de, gesehen am 20.6.2018.

37 Ein sprechendes Beispiel ist hier das frühe BlasenkammerSimulationsprogramm FAKE in der US-Teilchenforschung. Vgl. Gerald Lynch: Program FAKE: Monte Carlo Simulation of Bubble Chamber Events, University of California Lawrence Radiation Laboratory, Berkeley 1962, publications.|lbl.gou/islandoral object/ir\% 3 A137337|, gesehen am 29.6.2018. Dank an Arianna Borelli für diesen Hinweis.

38 Georg Jellinek: Allgemeine Staatslehre, Kronberg 1976 [1900], $338 \mathrm{ff}$.

39 Hannah Arendt: Die Lüge in der Politik, in: dies.: Wahrheit und Lüge in der Politik, München 2017, 7-42, hier 8.

40 Ebd., 19.

41 Hannah Arendt: Wahrheit und Politik, in: dies.: Wahrheit und Lüge, 44-92, hier 55 .
Produktion ein, während an der Geschaffenheit von Fakten ohnehin kein Zweifel bestehe. ${ }^{36}$

Damit spricht das Beispiel mediale Verfahren an, welche die Unterscheidungslinien zwischen Virtuellem und Materiellem zunehmend verschwimmen lassen: Wie im Beitrag von HANNAH ZINDEL diskutiert wird, betrifft dies z.B. epistemologische Transformationen im Kontext von (Computer-) Simulationen, die mit einer Fülle von <alternativen Fakten>, ungesicherten Annahmen oder gar kontrafaktischen Szenarien umgehen. ${ }^{37}$ Ebenso tangiert werden begriffliche Demarkationslinien zwischen Daten und Fakten, die im cross-referencing von Big Data und durch Fragen nach ihrer Ästhetisierung in neuartige Überlappungs- und Austauschbewegungen gebracht werden. Und darüber hinaus könnte man den Analyserahmen derartiger synthetischer Verfahren auf Bereiche wie ${ }_{3} \mathrm{D}$-Druck oder rapid prototyping ausdehnen, wo neue Objekte direkt aus digitalen Entwurfsumgebungen heraus entstehen. Spätestens dort zeigt sich auch eine ontologische Verschiebung an: Neben die vielzitierten Effekte einer «normativen Kraft des Faktischen ${ }^{38}$ treten neuartige Faktizitäten des Normativen.

\section{Praktiken/Politiken: Imagepflege als Weltpolitik und die Krise des Medialen}

Die Beziehung von Wahrheit und Politik wird historisch nicht nur als Begleiterscheinung politischer Krisen diskutiert, sondern steht vor allem dann zur Debatte, wenn das Politische selbst in die Krise gerät. Wie Hannah Arendt I97 I konstatiert, habe zwar die «Wahrhaftigkeit niemals zu den politischen Tugenden» gehört und die «Lüge immer als ein erlaubtes Mittel in der Politik» gegolten. ${ }^{39}$ Jedoch seien mit dem 20. Jahrhundert neue Akteur_innen hinter den Kulissen der politischen Bühne erschienen, mit welchen sich das Verhältnis von Wahrheit, Lüge und Politik erneut verschiebe. So gehe es in der politischen Debatte nicht mehr ultimativ um den Streit verhärteter Fronten, sondern vornehmlich um «Image-Pflege». ${ }^{\mathbf{4 0}}$ Tatsachenwahrheiten - eigentlich unumstößlich in der Realität verankert - würden zu Meinungen degradiert und nichts weniger als «die faktische Wirklichkeit selbst» ${ }^{\mathbf{4 1}}$ stehe dabei auf dem Spiel. Während sich Arendts Kritik zu Beginn der siebziger Jahre auf die um sich greifende Beschäftigung von PR-Berater_innen und «Problem-Lösern aus den Denkfabriken» kapriziert, welche sowohl die Politik selbst als auch die Medien durchdrangen, sind es heute gerade jene Medien, die im Fokus der Kritik stehen. Aus der Krise des Politischen wird auch eine Krise des Medialen. Die gegen- und wechselseitige Verbrämung sowohl klassischer <Qualitätsmedien〉 als auch zahlreicher Formate der <neuen Medien> als Fake News belebt nicht etwa einen produktiven, politischen Streit als Ausweg aus der Krise des Politischen, sondern alimentiert eine Diskussion, die sich mehr um die Medialität von Wahlkampf als um politische Inhalte 
dreht. Doch genau hier gerät das Mediale in die Krise, ist es doch seit jeher eine genuine Eigenschaft von Medien gewesen, sich im Hintergrund zu halten. Wo die Medien nur noch sich selbst zum Inhalt haben, scheint für das Politische kein Platz.

Projekte wie Forensic Architecture ebenso wie das von ASTRID DEUBERMANKOWSKY in dieser Ausgabe als aktuelles Beispiel einer Ecology of Practice beschriebene künstlerische Projekt von Alexandra Juhasz stellen Versuche dar, mithilfe medialer Praktiken und Technologien neuartige Informationsumgebungen zu schaffen, um sowohl die Krise des Medialen als auch die Krise des Politischen zu durchbrechen. Wahrheitsarbeit, wie sie Bertolt Brecht I93 I in der wichtigen Exilschrift «Über die Wiederherstellung der Wahrheit» ${ }^{42}$ als verpflichtende Übersetzungsarbeit eines jeden Einzelnen beschrieben hat, wird zu einer gemeinsamen Arbeit am Faktischen. Als kollaboratives Medienprojekt macht sich diese Arbeit die ständige Überarbeitung und Revidierbarkeit digitaler Medien nutzbar und weist Wahrheit somit einen praxeologischen Charakter zu. Den zunehmenden Angriffen nicht nur auf einzelne Tatsachenwahrheiten, sondern auf die Methoden und Mittel der Faktenproduktion allgemein, so Eyal Weizmann, müsse man dabei mit einem verstärkten «fact-based activism $»^{\mathbf{4}}$ unabhängiger Agenturen und Gruppen begegnen. Ebenso wie der Abdruck oder die Fotografie einstmals sowohl zu einer Krise der Kunst als auch zu einer Steigerung der Evidenzkraft des Medialen beigetragen haben, machen sich neue Medien nun das konstruktive und manipulative Element digitaler Technologien zunutze. Das Ziel dabei ist pragmatisch: nicht der Beweis einer vor den Dingen liegenden, höheren Wahrheit, sondern eine fortwährende Handwerksarbeit zur Herausstellung und Nutzbarmachung des Faktischen. Dass dieses Faktische durchaus auch mit Praktiken des Fakes zu ermitteln ist, führt schließlich der Beitrag von ANDREAS SUDMANN vor. Fake-Dokumentationen nutzen fingierte Geschichten, um auf dahinterliegende Realitäten zu verweisen. Inwiefern das Format darüber hinaus als Genre- und somit auch zur Repräsentationskritik dienen kann, wird von Sudmann jedoch in Frage gestellt.

Wie eingangs beschrieben befindet sich das mit diesem Schwerpunkt angesprochene Untersuchungsfeld nicht nur in ständiger Bewegung. Es ist auch gekennzeichnet durch unklare Ränder und Grenzen. Der vorliegende Themenschwerpunkt versteht sich daher als ein exploratives Unternehmen, bei dem es weniger um eine Bestandsaufnahme und Verengung auf vermeintlich Maßgebliches geht als darum, ein Forum zu bieten für tentative Erkundungen aktueller Faktizitäten. Die Art der hier versammelten Beiträge mag dieses Vorhaben unterstreichen. Anstelle <ausgefertigter>, streng fachwissenschaftlicher Artikel erreichten uns überwiegend essayistische, teils auch meinungsstarke Texte. Diese mögen in mancherlei Hinsicht ein wenig ungewohnt erscheinen im Vergleich zu vorangegangenen Ausgaben der ZfM. Wir halten sie dem Thema jedoch für unbedingt angemessen: Denn es geht
42 Vgl. Bertolt Brecht: Über die Wiederherstellung der Wahrheit, in: ders.: Gesammelte Werke, Bd. 20, Frankfurt/M., 1967, 191-198. 43 Branson: Forensic Architecture. 
uns im aktuellen Umfeld einer stark aufgeladenen Debatte um die Wechselverhältnisse von Politik, Wissenschaft und Medien zuvorderst darum, diese Debatte in den Beiträgen nicht nur beobachtet zu sehen, sondern den Autor_ innen auch Raum zu bieten, Möglichkeiten zur Intervention zu thematisieren und auszuloten.

Der Essay von BENJAMIN PETERS verknüpft dabei über eine Kritik des Theoriebegriffs aktuelle Diskussionen um Verschwörungstheorien mit Partikularisierungstendenzen in modernen Forschungscommunitys. Dazu zieht er sowohl Beispiele aus altgriechischer Wissenschaft wie aus aktueller US-Politik heran. Seine Problematisierung des Theoretisierens wird durch rezente Ereignisse nur noch stärker aufgeladen: Gerade haben z. B. Eltern von Opfern des Sandy-Hook-Highschool-Shootings den Verschwörungstheoretiker Alex Jones verklagt, der mittels seiner Alt-Right-Website Infowars.com und Radioshows die Theorie verbreitete, es habe sich bei dem Massaker um eine geschauspielerte Aufführung zur Durchsetzung schärferer Waffengesetze gehandelt - und es sei gar niemand dabei getötet worden. Derartige Behauptungen wurden - und hierin liegt die Brisanz - etwa anhand von Artefakten und technischen Unstimmigkeiten im zur Verfügung stehenden Videomaterial des Amoklaufs zu belegen versucht. Und es wurde dazu aufgerufen, auch hier im Sinne einer <citizen science> vorzugehen, mit dem Effekt, dass (trauernde) Anwohner_innen der Highschool mit den Nachforschungen <recherchierender> Jones-Anhänger_innen konfrontiert wurden. ${ }^{44}$ Was also lernt man über wissenschaftliche Theoriebildung, wenn man sie einer quasiwissenschaftlichen Evidenzproduktion im Bereich der Verschwörungstheorie gegenüberstellt? Oder kann andersherum ein paranoider Stil gar mögliche Erkenntniswege eröffnen? ${ }^{45}$

BERNHARD PÖRKSENS Beitrag schließt an derartige Vexierspiele an, erweitert den Fokus jedoch auf Fragen nach der soziopolitischen Verantwortung und der kommunikativen Rolle von Wissenschaft in Zeiten fragwürdiger Faktizitäten. Er widmet sich einem feuilletonistischen und teils auch akademischen Diskurs, der letztere als Effekt einer angeblichen Beliebigkeit der Postmoderne und eines Relativismus des Konstruktivismus skandalisiere und im Umkehrschluss eine Hinwendung zu neuen Realismen und Essenzialismen predige. Darin äußere sich jedoch nicht nur eine Selbstüberschätzung der Reichweite wissenschaftlicher Spezialdiskurse, sondern vor allem eine Perpetuierung von Idiosynkrasien - mit tragischen Folgen für durchaus mögliche und wirksame Interventionen von Wissenschaft und Universität gegen

44 Vgl. z. B. Matthew Haag: Sandy Hook Parents Sue Alex Jones for Defamation, in: The New York Times, dort datiert 17.4.2018, nyti. $\mathrm{ms} / 2 \mathrm{~J} Q$ PuaA, gesehen am 20.6.2018. 45 Vgl. z. B. Ned Rossiter: Paranoia is Real: Algorithmic Governance and the Shadow of Control, in: Media Theory, Vol. 1, Nr. 1: Manifestos, 2017. die hybriden Politikstile heutiger Autokraten, für die Skepsis und Wahrheitszweifel lediglich ein Instrument zur Durchsetzung eigener Interessen und Ideologien seien.

$\mathrm{Ob}$ diese Diagnose jedoch nicht ihrerseits die (angeblich) unterbleibende Selbstreflexion akademischer Diskurse durchaus übertreibt und dabei gerade die Rolle von Medientechniken unterschätzt, befragt CLAUS PIAS in einem 
Kommentar zu Pörksens Beitrag. Hier mag sich Stoff für eine weiterführende Debatte entwickeln, die sich - und das wäre ganz in unserem Sinne - auf die ZfM-Website verlängern könnte.

An der Schnittstelle von Theoriediskurs und medientechnischen und medienpraktischen Rückbezügen arbeitet auch der Beitrag von ASTRID DEUBERMANKOWSKY. Sie entwirft entlang des Projekts «\# Ioohardtruths-\#fakenews: A primer on digital media literacy» von Alexandra Juhasz mögliche Antworten auf die Frage, wie ein Denken mit dem Internet in und nach Fake News möglich ist. Juhasz begegnet den sogenannten alternativen Fakten der US-Regierung während deren ersten hundert Amtstagen mit jeweils hundert Wahrheiten über das Internet und wählt damit eine konstruktive Auseinandersetzung mit den Möglichkeitsbedingungen statt einer direkten Konfrontation mit der Fabrikation von Falschmeldungen. Die Ausbildung derartiger Informationsumgebungen diskutiert Deuber-Mankowsky mithilfe von Isabell Stengers Ökologie der Praktiken. Stengers bestimmt Denken als eine Praxis, die eine Relation zwischen Gehören-zu (belonging) und Werden (becoming) stiftet. Eine Ökologie der Praktiken weise insofern einen Ausweg aus dem Diskurs der Fake News, da sie helfe, Denkgewohnheiten zu ändern und den Praktiken so ein neues Habitat bereitzustellen - ein Habitat, wie es sich exemplarisch in Juhasz' Online-Projekt entwickelte.

In seinem Beitrag zum Genre der Fake-Dokumentation setzt sich ANDREAS SUDMANN mit einem weiteren ästhetischen Zugang zu Fragen des Faktischen auseinander. Fake-Dokus erweisen sich hier als zweifach interessant: Zum einen werden sie gemeinhin als hybride, repräsentationskritische Erzählform zwischen den Polen des Spiel- und Dokumentarfilms verstanden, die mit Mitteln der (übertriebenen) Konstruktion auf die Gemachtheit des Mediums Film hinweist. Andererseits hinterfragt der Beitrag jedoch diese verbreitete Zuschreibung kritisch und zeigt auf, wie sich jene häufig als repräsentationskritisch qualifizierte Form des filmischen Erzählens strukturell aufhebt und damit systemisch nicht unbedingt zu einer Krise der Repräsentation im Sinne eines deleuzianischen Projekts, sondern zur Krise der Repräsentationskritik selbst beiträgt.

Zuletzt untersucht HANNAH ZINDEL frühe Stadtklimastudien in Windkanälen und zeigt dabei auf, wie in den r96oer und s970er Jahren mit einer Wissenspraktik operiert wird, die sich als analoge Simulation bezeichnen lässt. Mit anderen Formen der Simulation teile diese eine konstitutive Ungenauigkeit: Ihre Ergebnisse figurierten eine veränderte Form von Faktizität jenseits binärer Unterscheidungen von wahr und falsch. Als Medientechniken der Verarbeitung und Erzeugung von Stadtklimadaten und als Medientechniken der Planung urbaner Gestaltung implementieren Windkanäle Zindel zufolge den epistemischen Status analoger Simulationen in einer zunehmend institutionalisierten Stadtklimaforschung und prägen deren Wissen über das Verhältnis von Stadt und Klima eminent mit. 
46 Vgl. Nassehi: Zu Fakten gibt es oft eine Alternative.
Und damit lässt sich jene noch umfassendere epistemologische Umstellung nachvollziehen, die den in diesem Schwerpunkt angesprochenen Status von Faktizitäten im Spannungsfeld von Medien, Wissenschaft und Politik betrifft. Denn vielleicht steht tatsächlich nicht mehr so sehr die moderne Unterscheidung von Erkenntnis und Erkenntnisgegenstand zur Debatte, sondern sind die Effekte neuer Medientechnologien zu diskutieren, die nicht mehr auf Objekterkenntnis, sondern auf Mustererkennung als vorherrschende Erkenntnisform setzen. Es handelt sich, so Armin Nassehi, um Informationsformen, die ihre zugehörigen Fragen erst während der Auswertung von Daten generieren, die nicht für die entsprechende Fragestellung erhoben wurden. ${ }^{46}$ Und damit wüssten wir nicht mehr nur alles, was wir wissen, durch Medien. Sondern Medien wüssten auch das, was wir wissen wollen könnten, von dem wir aber nicht wissen können. Daher gilt es umso mehr, das Fragen zu wagen.

EVA SCHAUERTE, SEBASTIAN VEHLKEN 